\title{
Simulation of granular soil behaviour using the Bullet physics library
}

\author{
E. Izadi \\ Laboratory of Geotechnics, Ghent University, Belgium
}

\author{
A. Bezuijen \\ Laboratory of Geotechnics, Ghent University, Belgium \\ Deltares, Delft, The Netherlands
}

\begin{abstract}
A physics engine is computer software which provides a simulation of certain physical systems, such as rigid body dynamics, soft body dynamics and fluid dynamics. Physics engines were firstly developed for using in animation and gaming industry; nevertheless, due to fast calculation speed they are attracting more and more attention from researchers of the engineering fields. Since physics engines are capable of performing fast calculations on multibody rigid dynamic systems, soil particles can be modeled as distinct rigid bodies. However, up to date, it is not clear to what extent they perform accurately in modeling soil behaviour from a geotechnical viewpoint. To investigate this, examples of pluviation and vibration-induced densification were simulated using the physics engine called Bullet physics library. In order to create soil samples, first, randomly shaped polyhedrons, representing gravels, were generated using the Voronoi tessellation approach. Then, particles were pluviated through a funnel into a cylinder. Once the soil particles settled in a static state, the cylinder was subjected to horizontal sinusoidal vibration for a period of 20 seconds. The same procedure for sample preparation was performed in the laboratory. The results of pluviation and vibration tests were recorded and compared to those of simulations. A good agreement has been found between the results of simulations and laboratory tests. The findings in this study reinforce the idea that physics engines can be employed as a geotechnical engineering simulation tool.
\end{abstract}

\section{INTRODUCTION}

In geotechnical engineering, most of the problems are simulated by means of continuum approaches (e.g. finite element method). In these approaches, soil is considered as a continuous homogeneous medium with certain mechanical properties. Although the use of continuum approaches are prevalent in geotechnical engineering practice, these fail to capture some of essential soil behaviour characteristics at micro scale, e.g. rolling of particles over each other or situations with large shear strains. They may provide acceptable results for engineering practice purposes, but they are not recommended for the study of soil behaviour at micro scale. Therefore, to account for such characteristics of soils in simulations, distinct element methods have been firstly occupied in geotechnics by Cundall \& Strack (1978, 1979). Distinct element methods have been successful in capturing the characteristics of soil which continuum methods were not able to take into account (O'Sullivan, 2011). But the usefulness of such approaches is limited because of long computation time as reported by Sakai et al., (2014) and O'Sullivan (2011). The more particles included in the model, the longer the calculation time will be. In addition, in distinct element methods, simplifications are normally applied in the geometry of soil particles. For instance, soil particles are represented by spherical objects; as a consequence, the influence of angularity of soil particles on the soil mass behaviour is neglected.

Recent developments in the field of hardware and software technology have led to systems capable of performing simulations including many objects (even objects with irregular shapes) and interactions among them in a relatively short time. These systems make use of sharing computational load on both central processing unit and graphics processing unit, making them extremely fast. Such systems are often called physics engines. Physics engines have been developed initially for evolvement of video games, animations and special effects in movies. Special motions of bodies and fluids, which are too complex to be manually handled by animators and game developers, can be handled and simulated realistically by physics engines. For example in the scenarios involving explosion, fracture or pouring of liquid on a surface, they play an important role in animations and video games (Bender et al., 2013; Akinci et al., 2012; Müller et al., 2013).

As mentioned before, physics engines are primarily developed for entertainment for games and movies, however, they have been used by researchers 


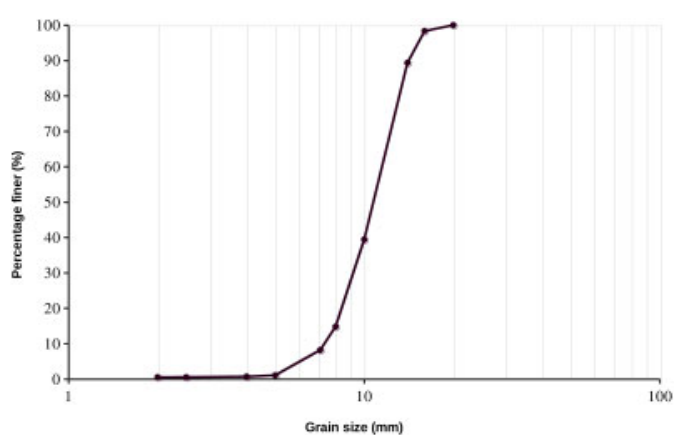

Figure 1. Grain size distribution curve.

for simulations in the fields of robotics (Richard, 2008; Basri et al., 2012) and industrial engineering (Servin \& Lacoursiere, 2008) too. But they have been rarely used in the field of civil and geotechnical engineering and even in those rare cases, they were employed merely for visualisation purposes rather than engineering analysis. Xu et al., (2013) conducted a finite element (FE) study on the bridge collapse accidents using element deactivation method. They integrated a physics engine into their FE analysis to numerically visualise the deactivated elements (the fragments detached from bridge structure due to failure) in their simulations. They reported that the integration of physics engine into FE analysis demonstrated a realistic and real-time visual simulation of the bridge collapse accidents. In a recent study, Cicekci et al., (2014) employed a game engine to merely visualise 3D profile of soil layers using borehole data. No literature was found in which the accuracy of physics engines is investigated in terms of quantitative engineering values in the field of geotechnical engineering. If physics engines prove to be fast and accurate simulation tools, it will be a step forward to advanced simulations in the field of geotechnical engineering.

The purpose of this study is to evaluate the accuracy of the physics engine called Bullet Physics Library (also called Bullet) in modeling of geotechnical engineering problems. To do so, series of pluviation and vibration tests were conducted on gravels in the laboratory and the obtained results were compared with those yielded from simulations. Simulations were carried out using distinct element based approach (also called rigid body dynamics). Special attention was given to the proper geometry of the simulated gravels and the preparation of soil samples.

\section{EXPERIMENTAL PROGRAMME}

All tests were conducted on poorly graded gravels (USCS: GP). Figure 1 shows the grain size distribution curve of the gravels. Since the gravels were made from crushed limestone, they have a blocky angular shape. Specific gravity of the gravels was equal to $2673.9 \mathrm{~kg} / \mathrm{m} 3$ and determined by Mercury Intrusion Porosity (MIP) test under zero pressure. The MIP test

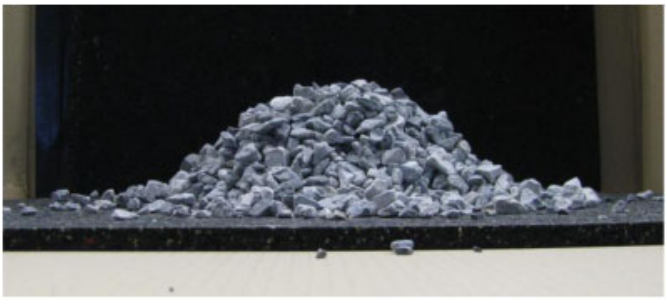

Figure 2. Profile of a pile of gravels resulted from a 'slow' pluviation test.

was done under zero pressure intentionally because the purpose was to determine the specific gravity at natural water content, without considering the pores inside the gravels. The gravels were thoroughly washed prior to sample preparation. In addition, through performing several slow pluviation tests on a rough surface, an angle of repose of 38.40 degrees was determined for the gravels. The term 'slow' indicates that the pluviation was done slowly to avoid dynamic effects on the gravel pile as well as the angle of repose. An example of pile of gravels is illustrated in Figure 2.

For the pluviation tests, the gravels were first mixed together thoroughly and then poured into a funnel with a lid on the bottom opening. Then, lid was removed and all the gravels fell into a cylindrical container with transparent sidewall and diameter and height of 11.5 and $20 \mathrm{~cm}$, respectively. The distance between the bottom of funnel and the top of the cylinder is denoted as falling height $(\mathrm{FH})$. Four cases of pluviation with FH of $0,5,10$ and $20 \mathrm{~cm}$ were performed in the laboratory experiments. It should be noted that, $\mathrm{FH}$ was kept constant during each test, i.e. the position of funnel and cylinder was fixed during each test. Upon completion of the pluviation tests, the standing height of the gravel was measured and recorded. Totally, 49 pluviation tests were carried out.

Vibration tests were conducted using an oscillator, capable of inducing sinusoidal horizontal waves with adjustable frequency and amplitude. All the vibration tests were performed on the samples which were prepared by the pluviation test described before with $\mathrm{FH}$ of $5 \mathrm{~cm}$. The cylinder of gravels was placed and restrained on a thick plate (called bottom plate). The bottom plate was placed on $252 \mathrm{~d}$ roller supports already arranged in a $5 \times 5 \mathrm{~cm}$ grid to ensure a full support for bottom plate and avoid any tilting during vibration. The setup used for vibration tests is shown in Figure 3. In order to avoid excessive bouncing of gravels and facilitating measurement of settlement due to vibration, a circular plate (called top plate) placed on top of gravels inside the cylinder before pluviation test. The diameter, thickness and weight of top plate were $11.3 \mathrm{~cm}, 1 \mathrm{~cm}$ and $104 \mathrm{gr}$, respectively. The vibration tests were performed in two different modes: in the first mode, the cylinder was vibrated for $20 \mathrm{sec}-$ onds (continuous vibration) and the displacement of the center of the top plate was measured before and after vibration; and in the second mode, cylinder was 


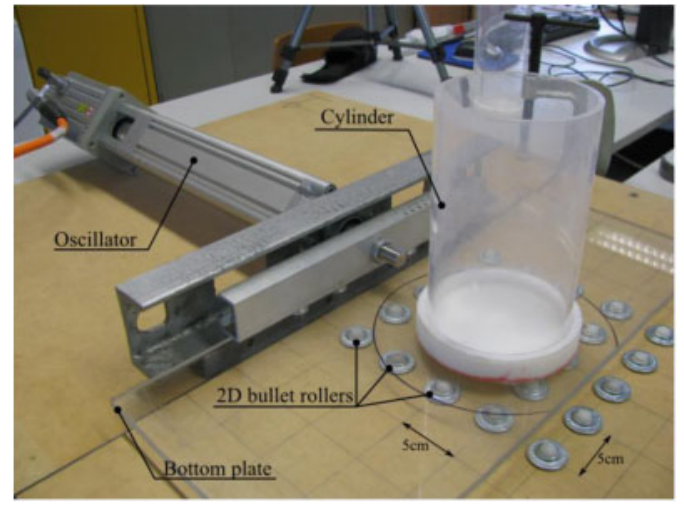

Figure 3. Equipment used for the vibration tests.

vibrated for four periods of 5 seconds (intermittent vibration), and the displacements of top plate after each vibration period were measured. For both modes, frequency and amplitude of vibration was kept to $3 \mathrm{cps}$ and $2.5 \mathrm{~cm}$, respectively. A total number of 33 vibration tests were performed. The gravel had a total mass of $2343 \mathrm{gr}$ in both the pluviation and the vibration tests. It is important to note that due to the large size of the gravels, scattering in the prospective results was expected at first. Therefore, a considerable number of tests were performed both for pluviation and vibration to have a better understanding of the range of obtained data.

\section{SIMULATIONS}

Bullet is an open source physics engine capable of performing collision detection, soft body and rigid body dynamics. Bullet has been integrated in Blender software making it easier for modeling, analysis as well as rendering, all in one platform. All the simulations were performed using the Bullet plugin in Blender software by means of rigid body dynamics module.

In the solver of the rigid body dynamics module, there is a simulation loop consisting of collision detection, collision resolution and time integration parts (Coumans, 2012). Collision detection deals with finding contact pairs among various bodies. In the collision resolution part, the contact normal and frictional forces and moments are resolved and subsequently, the positions and velocities of the bodies are updated in a specified time span in the time integration section. This loop is continuously followed until the end of the simulation. The rigid body dynamics solver computes the motions of bodies based on the NewtonEuler equations for both translational and rotational motions, in addition, joints and contacts are handled using relevant constraint/contact equations. Further details on equations and methods of solution can be found in the literature (Bender et al., 2012, Erleben et al., 2005).

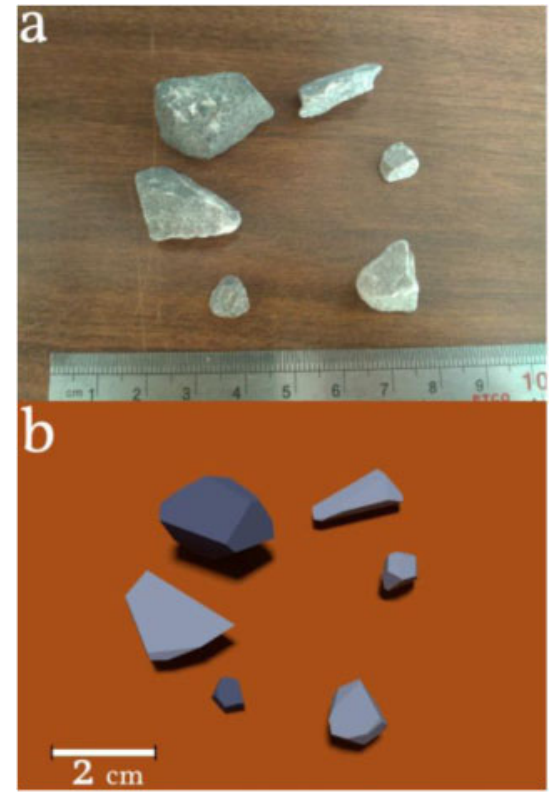

Figure 4. (a) Visual comparison of real gravels and (b) simulated ones using the Voronoi tessellation.

In order to create the geometry of gravels realistically, the Voronoi-based tessellation was used. Using this approach, blocky angular polyhedrons are created which represent the real gravels used in the laboratory tests quite well. A visual comparison of geometry of real gravels and simulated ones is shown in Figure 4. To model polyhedrons in a realistic size range, the data of the sieving test was used. Having the total mass remained on each sieve and specific gravity of gravels, the volume of gravels which remained on each sieve is obtained. Then, for each sieve a cube is simulated with the same volume as the volume of gravels remained on it. The average size of polyhedrons to be generated from the mentioned cube was set by the average mesh openings of the two successive sieves. After that, the Voronoi tessellation was performed on each block to generate polyhedrons. In reality, each sample with the mass of 100 gr contains 55 to 65 gravel stones. In the simulations a total number of 1456 polyhedrons were simulated which conforms well to the total mass of $2343 \mathrm{gr}$ was considered for the laboratory tests. This verifies the approach used for the gravel generation.

Based on the MIP test results, the density of the generated gravels was set to $2673.9 \mathrm{~kg} / \mathrm{m} 3$. In the rigid body dynamics module there is parameter called "collision margin" which defines the offset between the visualised surface of the object and the surface considered in the physics engine for collision detection and contact resolution (physical surface). To avoid any changes in the physical size of the gravels, this parameter was set to zero. The coefficient of restitution, which defines the magnitude of dissipated energy for each object when collides, was set to 0.315 based 


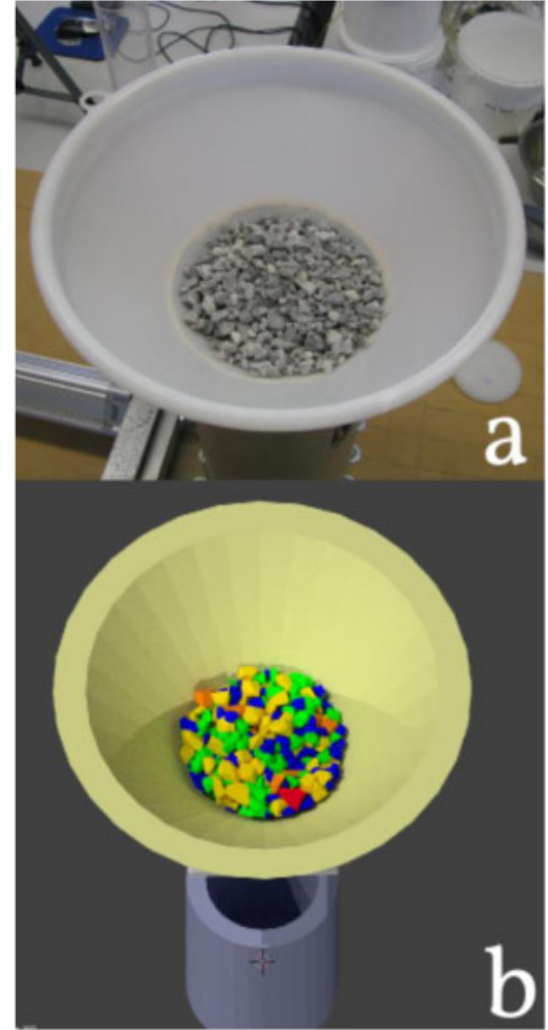

Figure 6. Gravels in the funnel prior to pluviation in the lab (a) and in simulation (b).

on the report of Robotham et al., (1995) for limestones, and a value of 0.793 was assigned to the friction ratio between the surface of gravels. The friction ratio was determined by the results of the slow pluviation tests performed to determine the angle of repose. As Lambe \& Whitman (1979) firstly proved and later was confirmed by Holsapple (2013), the angle of repose of the pile is the same as the angle of friction of the granular material when granular material is slowly pluviated on a rough surface in such a way that dynamic effects are not dominant. The effect of surface roughness of the gravels on the overall behaviour of granular flow is taken into account by the friction ratio adopted using this approach. The funnel, lid and cylinder were modeled as perfectly rigid objects and shown in Figure 5.

Prior to a simulated pluviation experiment, the simulated gravels were mixed thoroughly and then poured into the funnel (Figure 5). A lid was modeled right underneath the funnel opening to prevent gravels to fall through the funnel. Once, all the gravels settled in the funnel, the lid was removed and the gravels were allowed to fall through funnel into the cylinder. Like laboratory tests, pluviation was conducted with various falling heights and the height of standing gravels was recorded afterwards. In order to be able to investigate the reproducibility of the simulation results, three

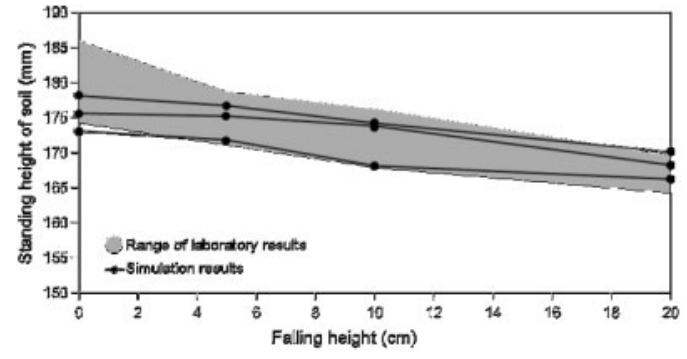

Figure 7. Laboratory and simulation results of pluviation tests.

different initial configurations for gravels, prior to pluviation, were used. The different configuration settings of gravel were obtained by additional mixing.

Like what was performed in the laboratory tests, the samples with $\mathrm{FH}$ of $5 \mathrm{~cm}$ were subjected to vibration tests. The top plate was allowed to fall slowly on the gravels inside cylinder to avoid any dynamic effects and extra settlement and then the height of top plate was measured. In all the vibration tests, frequency and amplitude were set to $3 \mathrm{cps}$ and $2.5 \mathrm{~cm}$, respectively. Continuous and intermittent vibrations were simulated according to the procedure explained in the experimental programme section and the position of the top plate was monitored constantly before, during and after vibration. The obtained results will be presented in the next section.

\section{RESULTS AND DISCUSSION}

The results of laboratory experiments and simulations are depicted in Figure 7. The gray shaded area in Figure 7, represents the range of standing height of soil in the cylinder for the laboratory tests. As mentioned before, a number of tests were carried out to determine this range. It can be seen that as the pluviation height increases, the height of soil in the cylinder decreases, comparable to the trend Raghunandan et al., (2012) reported for pluviation test on sands. The results of the simulations, for all three configurations, were within the range of laboratory results. It can be deduced that Bullet is capable of modeling the behaviour of pluviated gravels very well. In addition, it is observed that the results of the simulations are reproducible with regard to various gravel configurations.

Gravel configurations inside the cylinder, prior and after vibration, for both the laboratory tests and the simulations are illustrated in Figure 8. Note that the cylinder is deactivated from the $3 \mathrm{~d}$ viewport in Figures $8(\mathrm{c})$ and $8(\mathrm{~d})$. It can be seen from Figure 8, that in both laboratory tests and simulations, a denser configuration of gravels is achieved after vibration. Moreover, downward displacement of top plate is quite evident.

The results of continuous vibration tests are depicted in Figure 9. Since the measurement of the 

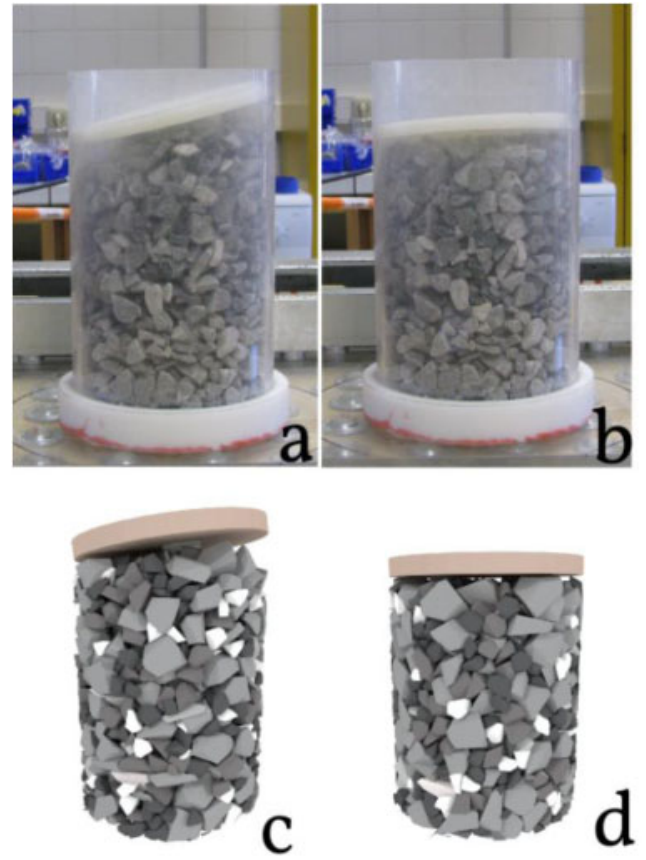

Figure 8. (a) Gravel configurations: in the laboratory before and (b) after vibration; and (c) in the simulations before and (d) after vibration.

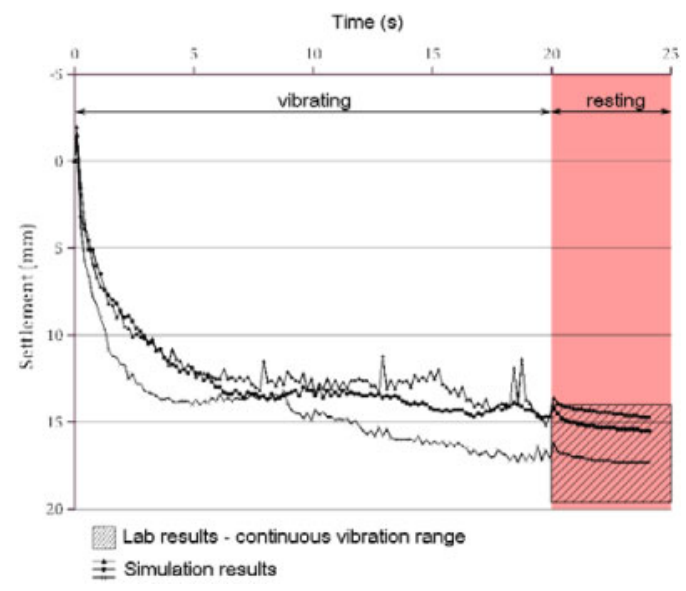

Figure 9. Top plate settlement due to continuous vibration.

position of top plate during vibration was not possible in the lab, only the range of final settlement is shown by the hatched area. The magnitude of settlement for the laboratory results ranges from 14 to $19.61 \mathrm{~mm}$. Conversely, in the simulations the position of top plate was continuously monitored every 0.08 seconds. As shown in Figure 9, in all the three cases, about $80 \%$ of settlement occurred in the first five seconds of vibration. The observed fluctuation of top plate position is due to the dynamic effects of gravels, especially the ones located on top layers. Depending on

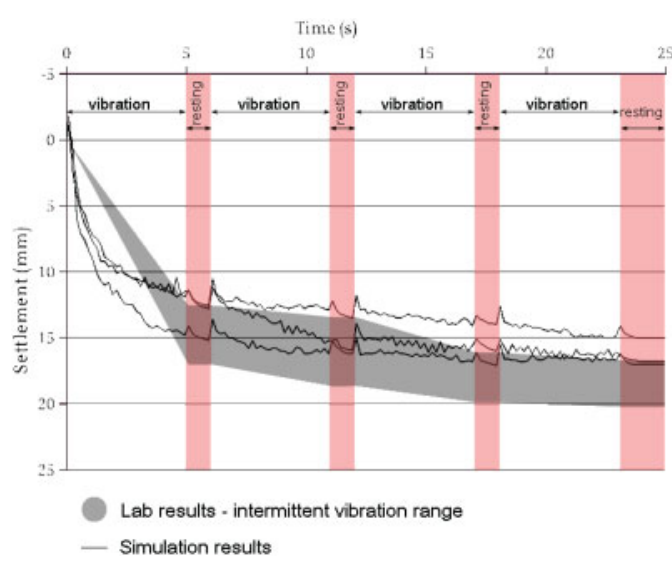

Figure 10. Top plate settlement due to intermittent vibration.

the interlock of gravels with each other, this fluctuation in settlement differs. The settlements shown by all three simulations are in a good agreement with the laboratory tests results.

Figure 10 demonstrates the results of intermittent vibration for laboratory tests and simulations. Note that in the laboratory tests the position of top plate was measured between two successive vibration periods. The laboratory results are shown using gray shaded area in the Figure 10 . Around $85 \%$ of total settlement occurred in the first vibration period both in the laboratory and simulations results. The simulation results conform well to the laboratory data, however, for one simulation case, a smaller magnitude for the settlement was observed. The yielded settlement for the mentioned simulation case is $11 \%$ less than the minimum range of laboratory results. Like continuous vibration, fluctuations in the position of top plate are seen, but major fluctuations are observed right at the end and at the beginning of each vibration period for the simulation results.

Note that a secondary sieve analysis was performed at the end of pluviation and vibration tests and it proved that no crushing occurred to the gravels during the tests.

\section{CONCLUSIONS}

This paper presented data relating to the behaviour of granular material when subjected to pluviation and vibration through laboratory experiments and simulations by the physics engine called Bullet. Special attention was paid to sample preparation as well as the shape of the gravel stones in the simulations. A good agreement has been observed between the results of laboratory tests and simulations, for both pluviation and vibration problems. The presented results in this study fortify the idea that physics engines can be employed as a geotechnical engineering simulation tool. 
The present study was a part of ongoing research on applicability of physics engines in simulation of geotechnical engineering problems. Further and more sophisticated tests and simulation are planned to be carried out in future.

\section{REFERENCES}

Akinci, N., Ihmsen, M., Akinci, G., Solenthaler, B., Teschner, M., 2012. Versatile Rigid-Fluid Coupling for Incompressibl SPH. Journal of ACM Transactions on Graphics (TOG), 31(4): Article no. 62.

Basri, N. M. H., Sahari, K. S. M., Mohideen, S. S. K., Roslin, N. S., Anuar, A., 2012. Development of 3d boiler header template using Webpots. Procedia Engineering, 41: 1463-1468.

Bender, J., Müller, M., Otaduy, M. A., Teschner, M., 2013. Position-based Methods for the Simulation of Solid Objects in Computer Graphics. State-of-the-art report, In STAR proceedings of Eurographics, Girona, Spain.

Bender, J., Erleben, K., Trinkle, J., Coumans, E., 2012. Interactive simulation of rigid body dynamics in computer graphics. State-of-the-art report, In STAR proceedings of Eurographics, Cagliari, Italy.

Cicekci, O. C., Turkeri, M. K., Pekcan, O., 2014. Development of Soil Profile Visualization Software Using Game Engines. Geo-congress: Geo-Characterization and Modeling for Sustainability, Atlanta, Georgia, February 23-26, 3364-3372.

Coumans, E., 2012. Bullet 2.80 physics SDK manual (Bullet physics library). Available from: http://bulletphysics.org/.

Cundall, P., Strack, O., 1978. The distinct element methods as a tool for research in granular media, Part I, Report to NSF.

Cundall, P., Strack, O., 1979. The distinct element methods as a tool for research in granular media, Part II, Report to NSF.

Erleben, K., Sporring, J., Henriksen, K., Dohlmann, H., 2005. Physics-based animation. Thomson Delmar Learning, Charles River Media Inc.
Holsapple, K. A., 2013. Modelling granular material flows: The angle of repose, fluidization and the cliff collapse problem. Planetary and Space Science, 82-83: 11-26.

Lambe, T. W., Whitman, R. V., 1979. Soil Mechanics, SI Version. New York: Wiley.

Müller, M., Chentanez, N., Kim, T. Y., 2013. Real time dynamic fracture with volumetric approxiamte convex decompositions. Journal of ACM Transactions on Graphics (TOG), 32(4): Article no. 115.

Raghunandan, M. E., Juneja, A, Benson Hsiung, B. C., 2012. Preparation of reconstituted sand samples in the laboratory. International Journal of Geotechnical Engineering, 6(1): 125-131.

Richard, V., 2008. Massively multi-robot simulation in stage. Swarm Intelligence, 2(2-4): 189-208.

Robotham, M. E., Wang, H., Walton, G., 1995. Assessment of risk from rockfall from active and abandoned quarry slopes. Trans. Inst. Mining and metallurgy A, 104(1-4): A25-A33.

O'Sullivan, C., 2011. Particulate discrete element modelling: A geomechanics perspective. New York: Taylor and Francis.

Sakai, M., Abe, M., Shigeto, Y., Mizutani, S., Tkahashi, H., Vire, A., Percival, J. R., Xiang, J., Pain, C. C., 2014. Verification and validation of a coarse grain model of the DEM in a bubbling fluidized bed. Chemical Engineering Journal, 224(15): 33-43.

Servin, M., Lacoursiere, C., 2008. Rigid body cable for virtual environments. IEEE Transactions on Visualization and Computer Graphics, 14: 783-796.

Xu, Z., Lu, X., Guan, H., Ren., A., 2013. Physics enginedriven visualization of deactivated elements and its application in bridge collapse simulation. Automation in Construction, 35: 471-481. 